\title{
Sustainability Aspects in Smart/Functional Textiles
}

\author{
Muhammad Zahid* \\ Istituto Italiano di Tecnologia, Italy
}

*Corresponding author: Muhammad Zahid, Smart Materials, Istituto Italiano di Tecnologia, Genova 16163, Italy.

\section{Received Date: July 12, 2018}

Published Date: August 14, 2018

\section{Editorial}

The consumer and industrial textile fabrics have grown to a multi-billion dollars market now a day, particularly, smart and functional materials (that are used for their extended functional features like water repelling, UV shielding, antibacterial etc.) are the current trend of consumer and institutional textiles and clothing. These smart and functional textiles have several applications in almost every field of life namely fashion, protection, medical, sports, uniforms, sensors and last but not the least packaging. By 2022 total market demand for functional textiles or, more generally, technical textiles have been anticipated to 35.5 million tons at an annual growth rate of $3.7 \%$. However, use of excessive artificial petroleum-based polymers, fibers, toxic chemical finishes and extensive consumption of energy and water in textile industries pose remarkable threats to the environment. For example, use of toxic solvents like chloroform is dangerous and causes Phosgene (a toxic gas) in the environment. Similarly, use of fluoropolymers in several textile finishes such as water repellent textiles induces bioaccumulative derivatives in the environment. As the environmental, social, and economic consequences associated with functional textiles are serious, health and the environmental regularity bodies, industries and researchers have responded to the concerns. Therefore, in the recent decade, many efforts have been dedicated to improving sustainability in the textile supply chain at each stage from raw materials to final finished garments for example. Sustainable textiles mean that all materials, process and finished goods are safe for both health and the environment, in all the phases of the product lifecycle. It also signifies biodegradability, bio-elimination and recycling aspects of these materials at the highest level of safety.

Consequently, many large manufacturers are taking initiatives and are ready to invest in sustainable processes as they have realized that it is also important to their own survival and growth in this competitive environment. Although many sustainable contributions can be made in the textile industry from pretreatment to the finished goods, lack of knowledge in the textile industry, availability of environmentally friendly chemicals, their cost, process scalability and technology transfer from research institution to the industry are major challenges that hinder in the adaptation of sustainable manufacturing. The efforts undertaken up to now are still insufficient to change the image of this industry; therefore, academic researchers have to play a major role to achieve these goals. There are some active areas where academic researchers can make a huge impact, for instance, develop textile finishing processes that are mainly aqueous based or don't involve toxic solvents like chloroform, find alternatives to fluorine based toxic chemicals for water repellent materials, exploit naturally available antibacterial materials instead of metallic nanoparticles and develop simple and easily scalable fabrication process. There are lots of initiatives already underway to produce overall sustainable textile products and the efforts are taken by responsible important players in the market and along the textile value chain, including textile machinery manufacturers too. However, it is still a long way to go and without active contribution of academic researchers and developers, it will not be easy to accomplish. 